\title{
A case of narcotic bowel syndrome successfully treated with clonidine
}

\author{
Voishim Wong, George Sobala and Monty Losowsky
}

Academic Unit of Medicine, St James's University Hospital, Beckett Street, Leeds LS9 7TF, UK

\begin{abstract}
Summary: Patients with chronic abdominal pain without an organic basis present a difficult management problem. Some of these patients may be prescribed opiates initially which may result in requiring progressively higher doses for pain relief. In this clinical setting, the suspicion of narcotic bowel syndrome should be borne in mind. With appropriate treatment and counselling, further invasive investigations including laparotomy may be avoided and resolution of symptoms can be achieved with clonidine. This case report demonstrates such a typical clinical scenario and discusses the possible aetiology and pathophysiology of narcotic bowel syndrome as well as the role of clonidine in controlling opiate withdrawal symptoms.
\end{abstract}

\section{Introduction}

Chronic abdominal pain is a common complaint both in general and hospital practice. ${ }^{1}$ Careful history, clinical examination and investigations generally result in a correct diagnosis and management plan. However, a proportion of patients with chronic abdominal pain are undiagnosable even after extensive investigations. They represent a difficult and challenging group of patients to manage. These patients may be prescribed narcotics for analgesic effect. Sometimes there is initial pain relief followed by an impressive worsening as tolerance develops which requires more opiates. This may result in a vicious circle of escalating pain followed by further narcotic administration for pain relief, which sets the scene for the development of narcotic bowel syndrome (NBS). ${ }^{2}$ We report a case of this clinical entity in which the pain resolved rapidly when narcotic administration was stopped. Opiate withdrawal symptoms were successfully alleviated with clonidine therapy.

\section{Case history}

A 40 year old woman was admitted for investigation of chronic intractable abdominal pain. The pain was colicky in nature with acute exacerbations

Correspondence: V. Wong, M.B., Ch.B., M.R.C.P., Level 7, Clinical Sciences Building, St James's University Hospital, Leeds LS79 7TF, UK

Accepted: 12 July 1993 which had gradually worsened over the last 10 years. Attacks were associated with nausea, 응 vomiting, anorexia, weight loss and constipation. She was taking a daily mixture of dihydrocodeine $6 \times 60 \mathrm{mg}$, intramuscular morphine $2 \times 15 \mathrm{mg}$ (given by her husband) and morphine sulphate (MST) $2 \times 20 \mathrm{mg}$ for 6 months. This odd regime had been prescribed by both her local general and hospital practitioners because of increasing intolerable abdominal pain. In the past she had had appendicectomy, hysterectomy and cholecystectomy for gallstones. She was also treated for pulmonary embolism with anticoagulation and given pain relief with coproxamol. Her symptoms recurred requiring repeated hospital admissions. Extensive investigations including inferior venocavogram and pulmonary angiography showed no evidence of recurrent pulmonary embolism and her anticoagulation was stopped 3 years later.

Her symptomatology of abdominal pain, nausea and vomiting began 10 years previously. Extensive investigations subsequently including two endoscopic retrograde cholangio-pancreatograms (ERCP) revealed no pathology. Her symptoms were thought to be suggestive of a functional bowel disorder, although diet changes and anti-spasmodics were tried unsuccessfully. Accordingly increasing frequency and dosages of opiatecontaining compounds were prescribed by her local general practitioner for pain relief. An emergency laparotomy was performed 4 years ago for symptoms and signs of intestinal obstruction which revealed only minor adhesions and a rotated 
transverse colon but no mechanical obstruction. She was then pain free for one year until her symptoms recurred. A further laparotomy showed adhesions in the right upper quadrant, a dilated duodenum and a duodeno-jejunostomy was fashioned for drainage. However, there was no improvement in her symptoms on this occasion.

On examination there were no remarkable physical findings other than previous abdominal scars. Haematological, biochemical, upper and lower endoscopic and imaging investigations excluded organic and rare metabolic causes (for example, acute intermittent porphyria) of abdominal pain. Barium meal and follow-through demonstrated the duodeno-jejunostomy anastomosis was patent but the small bowel transmit time was prolonged. An attempt was made to stop all her medication pending further tests. However, she experienced daily attacks of severe acute abdominal pain, nausea and vomiting which were only relieved with intramuscular diamorphine $15 \mathrm{mg}$ twice daily in addition to regular dihydrocodeine. During acute episodes, clinical examination revealed only right upper quadrant tenderness with no abdominal distension. Laboratory tests including liver function tests, amylase, porphyria screen and urinalysis were normal. Plain abdominal $\mathrm{X}$-rays revealed no obstructive feature. In the absence of other organic and biochemical causes of abdominal pain, a clinical diagnosis of NBS was made. Further opiate analgesics were stopped and clonidine hydrochloride $100 \mu \mathrm{g}$ twice daily was commenced. Over the next 3 days her pain subsided. She was discharged subsequently requiring no further opiate analgesia. She remained well and pain free 12 months later on follow-up.

\section{Discussion}

Chronic undiagnosable abdominal pain is a common clinical problem. It has been estimated that about $42 \%$ of males and $58 \%$ of females discharged from hospitals have no adequate explanation for their abdominal pain. ${ }^{1}$ The aetiology is often as elusive as the exact mechanism causing the pain. However, a proportion of these patients, particularly those on opiate medication, have narcotic bowel syndrome (NBS). NBS is a somewhat ill-defined clinical syndrome characterized by chronic recurrent abdominal pain secondary to prolonged opiate use or abuse. ${ }^{2,3}$ The pain is colicky in nature, rarely localized and typically accompanied by normal laboratory tests even during acute episodes. Nausea and vomiting are frequently part of the clinical picture. Weight loss is usually mild and secondary to anorexia, abdominal pain and fear of vomiting. Signs of pseudoobstruction can be present in some patients. These findings make the other differential diagnoses including primary biliary or pancreatic disease or renal calculi unlikely. Further investigations should be performed to exclude other possibilities such as peptic ulcer disease, gynaecological pathology and metabolic disorders. Our patient illustrates the extreme difficulties in making the diagnosis; extensive investigations at different hospitals over a time interval of over 10 years had failed to demonstrate an organic basis. This may also account for the fact that the actual incidence of NBS in the different patient groups is unknown. It may also be due to poor recognition as it has not even been formally recognized in the recent international categorization of functional gastrointestinal disorders. ${ }^{4}$ Nevertheless, it had been estimated in one study that as many as $65 \%$ of patients with chronic non-malignant pain were misusing drugs, mainly of the opiate group. ${ }^{5}$

The pathophysiology of NBS is poorly understood. However, the current evidence suggests that opiates, both endogenous and exogenous, act directly on the gastrointestinal tract by means of several types of receptors present in the gut wall and central nervous system. ${ }^{6,7}$ It is not entirely clear, however, whether opiates act via inhibition of acetylcholine release, modulation of dopaminergic effects, alteration in synthesis and release of prostaglandins, modulation of vasoactive intestinal polypeptide levels, or control of sympathetic outflow. ${ }^{8}$ The summation of effects of opiates in humans results in increased muscular tone or spasm of gastric antrum, small intestine and colon, ${ }^{9-11}$ and an increase in gut segmental contractions that are thought to be non-propulsive., ${ }^{9,12}$ They may also produce contraction of several enteric sphincters including the pylorus, sphincter of Oddi, ileocaecal sphincter and anal sphincter. ${ }^{8,10}$ In addition, opiates may directly increase net electrolyte and water absorption across the mammalian intestinal mucosa and decrease the volume of gastrointestinal water loss induced by various secretagogues. ${ }^{13}$ Thus the combination of altered gut motor activity, which allows prolonged contact between gut contents, and direct actions on the intestinal mucosa results in a reduction in stool volume and frequency and prolongs gut transit time. ${ }^{13,14}$ This may lead to profound constipation. The central effects of opiates may contribute by other ways such as reduced awareness of defaecatory stimuli. ${ }^{15}$ The net result of opiate administration is initial pain relief followed by delayed bowel transit leading to nausea, vomiting, constipation, and abdominal pain as distension and spasm become more pronounced. Pain relief is often temporary and inadequate, resulting in repeated use of narcotics at increasing dose and frequency for periods of months to years as in our patients. 
There are several possible explanations for the development of NBS. Firstly, the direct actions of narcotics on the gut over time may alter intestinal motility, gut reflexes and gastrointestinal sphincter activity as well as physical changes in gut luminal contents. This may produce the symptoms as described. Alternatively, prolonged opiate use could cause pancreatobiliary pain as a result of chronic constriction of the sphincter of Oddi. It has even been postulated that some of the symptoms of NBS could be due to localized withdrawal effect on intestinal motility well before the development of the characteristic generalized features of narcotic withdrawal. ${ }^{3}$ It must be emphasized that whether the symptom complex of NBS are due to actions of opiates on the gut or the withdrawal of opiates are not well understood.

Withdrawal from opiates is characterized by a hyperexcitable state in organs previously depressed. ${ }^{15}$ The symptomatology includes nausea, vomiting, diarrhoea and crampy abdominal pain in addition to restlessness, anxiety, yawning, muscular aching and pilo-erection. It is thought to be mediated by adrenergic mechanism. ${ }^{16}$ Administration of narcotics during this period abruptly halts the development of these withdrawal symptoms. Alternatively, clonidine can be used to abolish

\section{References}

1. Rang, E.H., Fairburn, A.S. \& Acheson, E.D. An enquiry into the incidence and prognosis of undiagnosed abdominal pain treated in hospital. Br J Soc Med 1970, 24: 47-51.

2. Roger, M. \& Cerda, J.J. Editorial: the narcotic bowel syndrome. J Clin Gastroenterol 1989, 11: 132-135.

3. Sandgren, J.E., McPhee, M.O. \& Greenberger, N.J. Narcotic bowel syndrome treated clonidine. Ann Intern Med 1984, 101: $331-334$.

4. Thompson, W.G., Creed, F., Drossman, D.A., Heaton, K.W. \& Mazzacca, G. Functional bowel disease and functional abdominal pain. Gastroenterology Int 1992, 5: 75-91.

5. Maruta, T., Swanson, D.W. \& Finlayson, R.E. Drug abuse and dependency in patients with chronic pain. Mayo Clin Proc 1979, 54: 241-244.

6. Burks, T.F., Galligan, J.J. \& Porreca, F. Gastrointestinal drug receptors. J Clin Gastroenterol 1983, 5(Suppl 1): 29-36.

7. Bianchi, G., Ferrenti, P., Recchia, M. et al. Morphine tissue levels and reduction of gastrointestinal transit in rats: correlation supports primary action site in the gut. Gastroenterol 1983, 85: 852-858.

8. Malagelada, J.R. Potential pharmacological approaches to management of gut motility disorders. Scand J Gastroenterol 1984, 19 (Suppl 96): 111-122.

9. Read, N.W. Speculations on the role of motility in the pathogenesis and treatment of diarrhoea. Scand J Gastroenterol 1983, 84 (Suppl): 45-63.

10. Daniel, E.E. Pharmacology of the gastrointestinal tract. In: Code, C.F. (ed.) Handbook of Physiology, Vol. 4. Williams and Wilkins Co., Baltimore, 1968, pp. 2267-2324.

11. Konturek, S.J. Action of enkephalins on the digestive system In: Bloom, S.R. \& Polak, J.M. (eds) Gut Hormones, 2nd edn. Churchill Livingstone, Edinburgh, 1981, pp. 432-440. these symptoms as has been utilized for many years successfully in drug rehabilitation. ${ }^{19,20}$ The exact mechanism of action is unclear. Clonidine, an alpha-2 adrenergic receptor agonist, is thought to act directly and indirectly via the stimulation of alpha-2 receptors in the locus coeruleus of the central nervous system and the gut wall., $, 17,18$ Administration of clonidine, in general, results in the reduction of the rate and amplitude of intestinal contraction and relaxation of the intestinal smooth muscle. ${ }^{8,18}$ The overall effect is the relaxation of the gastrointestinal tract. Clonidine therapy has been used successfully in patients with NBS to break the vicious circle of recurrent symptoms and narcotic requirement. ${ }^{3}$ The dosage is $0.1 \mathrm{mg}$ twice initially and increasing to $0.1 \mathrm{mg}$ four times daily if necessary. It should only be initiated under close medical supervision as side effects such as postural hypotension could occur. With this regime, our patient made an excellent response within 1 week and had remained so 12 months later.

Thus the diagnosis of NBS should be considered in any patient with chronic unexplained abdominal pain or pseudo-obstruction who is using or abusing opiates for pain relief. Early diagnosis may avoid unnecessary investigations and even laparotomy.

12. Powell, D.W. Muscle or mucosa: the site of action of antidiarrhoeal opiates? (Editorial). Gastroenterology 1981, 80: 406-408.

13. Turnberg, L.A. Antisecretory activity of opiates in vitro and in vivo in man. Scand J Gastroenterol 1983, 84 (Suppl): 79-83.

14. McKay, J.S., Linaker, B.D. \& Turnberg, L.A. Influence of opiates on ion transport across rabbit ileal mucosa. Gastroenterology 1981, 80: 279-284.

15. Jaffe, J.H. \& Martin, W.R. Opioid analgesics and antagonists. In: Gilman, A.G., Goodman, L.S. \& Gilman, A. (eds) Goodman and Gilman's The Pharmacological Basis of Therapeutics, 6th edn. Macmillan Publishing Co., New York, 1980, pp. 494-534.

16. Kuperman, D.A., Swinsky, C.A. \& Lynch, D.R. Myoelectric activity of the small intestine during morphine dependence and withdrawal in rats. Am J Physiol 1987, 252: G562-567.

17. Bakris, G.C., Cross, P.D. \& Hammarsten, J.E. The use of clonidine for management of opiate abstinence in a chronic pain patient. Mayo Clin Proc 1982, 57: 657-660.

18. Gold, M.S., Redmond, D.E., Jr \& Kleber, H.D. Clonidine blocks acute opiate withdrawal symptoms. Lancet 1978, 2 : 599-602.

19. Doherty, H.S. \& Hancock, A.A. Role of alpha-2 adrenergic receptors in the control of diarrhoea and intestinal motility. $J$ Pharmacol Exp Ther 1983, 225: 269-274.

20. Schreier, W.A. \& Burks, T.F. Suppression of morphine withdrawal diarrhoea by clonidine. Proc West Pharmacol Soc 1981, 24: 341-345. 